\title{
Research on the Path to Breakthrough in the Dilemma of Chinese Traditional Medicine Intellectual Property Protection
}

\author{
Hongyuan $\mathrm{Li}^{*}$ \\ Department of Law, School of Law, Humanities and Sociology, Wuhan University of Technology, Wuhan, Hubei, \\ China \\ *Corresponding author.Email:lihy720@163.com
}

\begin{abstract}
In the protection of intellectual property rights of traditional Chinese medicine in my country, there are problems such as insufficient awareness of enterprises and individuals to protect their intellectual property rights and difficulty in patent application. The current laws and regulations cannot guarantee the rights of these people. If we want to better protect the intellectual property rights of Chinese medicine in our country, we must insist on enhancing the awareness of intellectual property rights protection from the source, and further improve the patent formula protection mechanism, so as to provide comprehensive protection to the Chinese medicine industry from beginning to end. Only in this way can Chinese medicine develop better, and in the future we can enjoy better quality Chinese medicine products.
\end{abstract}

Keywords: Intellectual property, Traditional Chinese medicine, Development research.

\section{THE STATUS QUO OF INTELLECTUAL PROPERTY PROTECTION OF CHINESE MEDICINE}

As an important part of Chinese traditional culture for thousands of years, the protection of traditional Chinese medicine knowledge is related to the inheritance and protection of Chinese excellent culture. However, due to the characteristics of traditional Chinese medicine knowledge that are different from modern knowledge, and the current intellectual property system is mostly transplanted or referred to the Western intellectual property protection system, it has not realized the legitimate interests of the holders of traditional Chinese medicine knowledge.[1] The purpose also hinders the development of Chinese medicine.

\subsection{Lack of Awareness of Active Protection}

Due to the lack of effective legal protection, the intellectual property rights of Chinese medicine are often used by pharmaceutical companies in a few developed countries for free commercial use, and use the existing intellectual property system to obtain huge profits, and even restrict our use. If a company uses advanced technology to analyze the pharmacology and toxicology of traditional Chinese medicine prescriptions or classic prescriptions and make them into proprietary Chinese medicines, [2] according to the current drug regulatory system and intellectual property system, other companies can produce this product without the approval of the first R\&D manufacturer. This is bound to cause market chaos and at the same time make domestic companies lose interest in developing traditional Chinese medicine. If foreign companies borrow traditional Chinese medicine knowledge to develop and produce monomer medicines, or possess our traditional knowledge in other ways, the existing Chinese medicine prescriptions are not protected by trademark law[3] and intellectual property law because they are not industrialized. Many foreign companies have infringed on the intellectual property rights of Chinese medicine by various means. In the beginning, the effective ingredients were extracted from Chinese medicinal materials to circumvent the protection of intellectual property rights. Later, the trademarks of traditional Chinese medicine such as "Li Shizhen" and "Compendium of Materia Medica" were registered as trademarks; now, the provisions and prescriptions of 
classic Chinese medicine are copied. "Original taste" is used to promote sales and increase the cultural added value of products. For a long time, the domestic TCM industry lacked the awareness of intellectual property protection. Many TCM experts did not know which drugs and technologies belong to the scope of protection. Even if they knew, they did not know how to protect, sometimes even some mature hospital preparations. The formulas fall into the hands of others for nothing.

\subsection{Incomplete Intellectual Property Protection System}

At present, except for a few systems such as integrated circuit layout design, most of my country's intellectual property system can be applied to the protection of Chinese medicine knowledge. However, the current intellectual property system represented by patents and trade secrets is not yet perfect for the protection of Chinese medicine. The protection clauses on pharmaceutical patents in my country's patent law are based on the relevant content of the patent law of western countries on chemical medicines. Traditional Chinese medicine and chemical medicine belong to two completely different theoretical systems. The protection of chemical medicine is used to protect the intellectual property rights of Chinese medicine., It will inevitably be unsatisfactory. The current patent system requires that the objects of protection are novel, creative, and practical. Traditional medicine belongs to the category of traditional knowledge. Most of them are existing intellectual achievements and are in a public state. They are included in the patent system and have entered the public domain. The basic conditions for granting patent rights can be used freely and free of charge without permission from anyone. Trademark law generally does not protect specific technology or knowledge itself, and only when traditional medicine is industrialized, commercialized, and marketized, can the trademark system play a role. However, it is difficult to industrialize and market the vast majority of Chinese medicine knowledge, and therefore it is difficult to obtain the protection of trademark law. The objects protected by the copyright law cannot exceed a certain period of time, but the knowledge of traditional Chinese medicine is mostly passed on from generation to generation, and the protection period is too early to meet its protection requirements. Therefore, in order to solve this problem, we should appropriately modify and perfect our country's existing intellectual property legal system, so that it can take into account the actual needs of protecting traditional knowledge such as traditional Chinese medicine.

\section{INTELLECTUAL PROPERTY PROTECTION MEASURES FOR TRADITIONAL MEDICINE OUTSIDE THE TERRITORY}

\subsection{Traditional Medicine Protection in Japan}

A lot of knowledge of traditional Chinese medicine in Japan comes from our country. In theory, the number of patent applications for traditional Chinese medicine and the research and development of traditional Chinese medicine should be much higher than that of Japan, but the facts are just the opposite. After a lot of knowledge of traditional Chinese medicine spread to Japan, Japan is here. Based on further development and research, and then apply for patent protection as a domestic product, this phenomenon is directly related to the Japanese patent system.[4] China should learn from the outstanding aspects of the Japanese patent system and use it for our country. Knowledge of Chinese medicine developed in our country.

\subsection{Patent Conversion Application}

When applying for patents, many applicants may not pass the examination standards for invention patents during the examination process, but they can apply for utility model patents after the second is the most difficult application. My country and Japan have similar regulations. However, for applications from simple to difficult conversion, this is unique in Japan. According to the Japanese Patent Law, applicants can change their utility model patent applications into invention patent applications or pattern design registration applications within the time specified by the law. Change to patent application. Flexible application conversion has greatly increased the number of patent applications in Japan.

\subsection{Extension of Patent Protection Period}

In terms of the protection period of patents, the protection period of invention patents in my country and Japan are the same, both of which are 20 years. In terms of the protection period of utility models, the protection period of utility models is 10 years in my country and 15 years in Japan. In addition, Japan has loose examination standards for the extension of the patent protection period. As long as the right holders can apply for an extension of the protection period within three months of the approval of the relevant authorities as stipulated in the Japanese Patent Law, once the examination is qualified, The period can be extended for another 5 years. [5]The extension of the patent protection period once again brings huge economic profits to the right holders, thereby stimulating the innovation ability of the people and promoting the development of traditional medicine. 


\section{THE FUTURE DEVELOPMENT PATH OF MY COUNTRY'S TRADITIONAL CHINESE MEDICINE INTELLECTUAL PROPERTY PROTECTION}

\subsection{Establish Trademark Awareness and Build Well-Known Brands}

As the best salesman of products, trademarks even play a key role in the survival of enterprises. Trademarks are more important to traditional Chinese medicines than other products because consumers of other products can judge from the appearance of the products. The quality of the product is good or bad, and some can be used to understand the performance of the product, and for products that you are not satisfied with, you can also enjoy a seven-day refund for unreasonable returns, but these cannot be achieved and satisfied in the field of traditional Chinese medicine. Therefore, consumers are choosing In addition to following the doctor's advice when taking medicine, the most important thing is the trademark and brand. In addition, my country has many time-honored brands with a long history, good reputation, and high international reputation. Therefore, my country takes this opportunity to build a well-known brand of Chinese medicine and further enhance its international competitiveness. In this process, you should pay attention to the following items: highlight the trademark and dilute the generic name of the drug. In most cases, many owners are used to highlighting the generic name of the drug or sometimes use the generic name of the drug when registering. If the generic name of the drug is recognized as the current drug, the right holder will no longer own the trademark right, or the public's attention will be slightly reduced. This will bring great adverse effects to the enterprise.

\subsection{Pay Attention to Drug Patent Applications}

The reason why Chinese medicinal materials are not innovative enough is because the inherent protection methods hinder their progress. The breakthrough to improve product creativity lies in breaking the original protection method of "Ancestral Secret Recipe" and focusing on patent protection. Patent applications have three requirements, namely creativity, novelty and practicality. In order to apply for patent protection, Chinese medicine developers must meet these three requirements, especially creativity. Creativity requires outstanding features and substantive progress. For developers, it is an opportunity to stimulate innovation and creation. To make traditional Chinese medicine products more complete protection, they must meet the requirements of creativity. The improvement of creativity is not only the imagination of developers, but also the update of technological means makes them more effective. [6]The degree of product innovation is also a standard for patent protection. The transformation of traditional Chinese medicine from natural products to new medicines is an extremely cumbersome and complex process. This process contains countless links, and each link may produce new inventions. New inventions and creations mean that patent protection applications will also increase. [7]The size is directly proportional to the strength of patent protection. Therefore, if you want to get more complete protection, improving creativity is a necessary condition.

\section{CONCLUSION}

The legislature of our country should strengthen and improve the legislation on traditional Chinese medicine. In this regard, it should focus on listening to the opinions of two groups of people. One is the staff working in the field of traditional Chinese medicine, who understand the properties of medicines and the protection goals they want to achieve; One is the opinions of legal experts, who understand the boundaries of the law and how to ensure that Chinese herbal medicines are protected to the utmost extent. To form a relatively complete intellectual property protection system for traditional Chinese medicines, especially to strengthen the patent protection of traditional Chinese medicines, legislation should also list as much as possible the various situations of infringement of Chinese medicine patents, and detailed regulations must be made on the types and methods of infringement and the consequences of infringement. , To ensure that there are no details, so that there are laws and rules to follow when encountering Chinese medicine infringement cases.

\section{REFERENCES}

[1] Li Xiuming, Zhang Jing, Han Guixiang. Analysis of the cultural heritage of traditional Chinese medicine and the path of intellectual property protection under the background of independent innovation in the new era $[\mathrm{J}]$. China Medical Herald, 2020, 17(05): 152-155.

[2] Wang Lei, Lin Aicui, Sun Zhonghe, Wen Xing. Research on the Sustainable Protection of Ethnic Medicine under the Trend of Internationalization [J]. Herald of Chinese Medicine, 2020, 26(01): 41-43.

[3] Liu Changqiu. Problems and countermeasures of intellectual property protection of traditional Chinese medicine in my country [J]. Chinese Journal of Traditional Chinese Medicine, 2019, 34(11): 5011-5013.

[4] Chen Qing. Evaluation and System Enlightenment of Traditional Chinese Medicine Traditional Knowledge from the Perspective of Patent [J]. Intellectual Property, 2017(10):75-81. 
[5] Song Jian. On the Types of Right Subjects of Traditional Knowledge of Traditional Chinese Medicine-Also on the perfect ideas of my country's legislation [J]. Journal of Guizhou Normal University (Social Science Edition), 2019(05):152-160

[6] Dong Yanlin, Feng Xinxin, Wang Mingxu.
Connotation analysis of the protection of traditional Chinese medicine knowledge [J]. Chinese Health Service Management, 2018, 35(04): 272-274.

[7] Li Dongmei. The dilemma and countermeasures of intellectual property protection of traditional Chinese medicine [D]. Gansu University of Political Science and Law, 2019. 